\title{
Chronic Epididymo-Orchitis Mimicking Tuberculosis Turned Out to be Leprosy: A Case Report
}

\author{
Mukesh Chandra Arya ${ }^{1 *}$, Ankur Singhal ${ }^{2}$, Vivek Vasudeo ${ }^{2}$, Ajay Gandhi ${ }^{2}$, Rakesh Singh $^{2}$ and Mahesh Sonwal ${ }^{2}$ \\ ${ }^{1}$ Professor and Head, Department of urology, Sardar Patel medical college, Bikaner, Rajasthan, India \\ ${ }^{2}$ Department of urology, Sardar Patel medical college, Bikaner, Rajasthan, India
}

*Corresponding author: Mukesh C Arya, Professor and Head, Department of urology, Sardar Patel medical college, Bikaner, Rajasthan, India.

Received Date: October 14,2020

Published Date: November 23, 2020

\begin{abstract}
We report a 55-year male with a prior history of treatment for bilateral Epididymo-orchitis without relief. We thought of genitourinary tuberculosis (GUTB) or nonspecific Epididymo-orchitis as differentials. Suddenly he developed crops of lepromatous skin lesions. On reviewing the literature, we found the involvement of testes in leprosy in 60-90\% of cases. However, urology literature and textbooks do not mention leprosy to be a differential diagnosis of orchitis or chronic Epididymo-orchitis. Therefore, testicular atrophy, infertility or chronic Epididymo-orchitis should make us consider the possibility of leprosy, considering the half of the global burden in India.

Keywords: Chronic Epididymo-orchitis; Testicular atrophy; Orchitis; Leprosy; Infertility

Abbreviations: GUTB: Genitourinary Tuberculosis; ED: Erectile Dysfunction; ENL: Erythema Nodosum Leprosum; MB MDT: Multibacillary Multi

Drug Therapy; LUTS: Lower Urinary Tract Symptoms; UTI: Urinary Tract Infection; LH: Luteinizing Hormone; FSH: Follicle Stimulating Hormone
\end{abstract}

\section{Introduction}

The causes of chronic orchitis and Epididymo-orchitis are varied including, urinary tract infection (UTI), gonococcal or nongonococcal (chlamydia, ureaplasma) infections, genitourinary tuberculosis (GUTB), post-vasectomy and drug-induced. However, the Urology textbooks do not mention leprosy as a differential diagnosis in such cases [1]. Herein, we present a case of Epididymoorchitis caused by leprosy with a review of the literature. Leprosy is a chronic infectious disease caused by Mycobacterium leprae. Leprosy affects mainly the skin and peripheral nerves. Its diagnosis is established on the skin and neurologic examination of the patient. Involvement of testis and epididymis is well described in dermatology literature with an incidence ranging from $23.6 \%$ to $68.3 \%[2,3]$. Testicular involvement is more in lepromatous leprosy and may result in infertility and impotence. However, practicing urologist does not keep this condition as a differential diagnosis and many such cases might remain undiagnosed. There are several classification systems validated for leprosy. The most commonly used Ridley \& Jopling classification system (1966) is based on the concept of spectral leprosy and uses clinical, immunological, and histopathological criteria [4]. The spectrum consists of tuberculoid form at one end and the lepromatous form at the other end. The borderline form is divided into borderline-tuberculoid, borderlinelepromatous, according to the greater proximity to one of the poles, and borderline-borderline. Multi-Drug Therapy is the cornerstone of the treatment of leprosy.

\section{Case History}

A 55-year Hindu male, resident of Bikaner, Rajasthan, a farmer by occupation came with presenting complaints of scrotal swelling along with lower urinary tract symptoms (LUTS) and low-grade 
evening rise of temperature for 7 months. He initially consulted many clinicians and had treatment with antibiotics and antiinflammatory drugs without any improvement. He also had a loss of libido and erectile dysfunction (ED) (Sexual Health Inventory for Men score 8). He had completed his family with no history of extramarital contact. General physical examination was normal except low-grade fever. Local examination revealed left small testis (reduced sensation), bulky epididymis and right Epididymoorchitis with secondary hydrocele. There was no gynecomastia and he had male pubic hair pattern. Nonspecific Epididymo-orchitis and genitourinary tuberculosis were kept as differentials. He was investigated on these lines. His ESR was 38, total counts were 13000 with polymorphonuclear Leukocytosis. Uroflowmetry
(Qmax 18ml/sec) was normal. Ultrasonogram suggested Bilateral epididymitis and testicular atrophy with left-sided hydrocele. Ultrasound abdomen did not pick up any mesenteric or retroperitoneal lymphadenopathy. During his hospital stay, he developed crops of erythematous, raised, painful, nodules with ulceration. Biopsy of skin lesion was taken which was suggestive of lepromatous leprosy(Figure 1).

After reappraisal, he was found to be a defaulter of multibacillary leprosy two years ago. Even at this stage, we did not suspect it to be due to leprosy. On reviewing dermatology literature leprous involvement of testis was suspected. Biopsy of testis and epididymis were then taken, which showed evidence of leprosy (Figure 2 and 3).

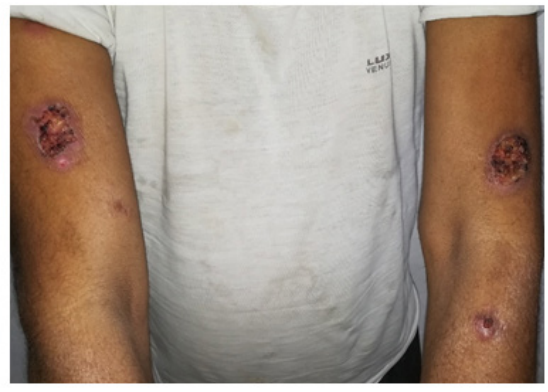

Figure 1: Skin lesions in both upper limbs.

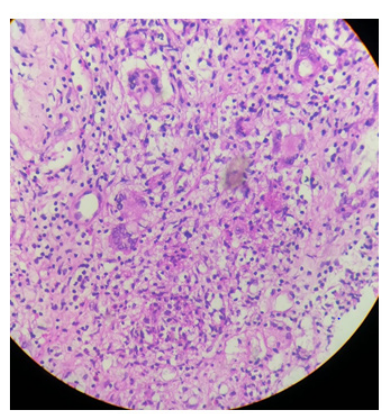

Figure 2: Zeihl-Neelson staining showing Acid Fast bacilli (Mycobacterium leprae) Low power.

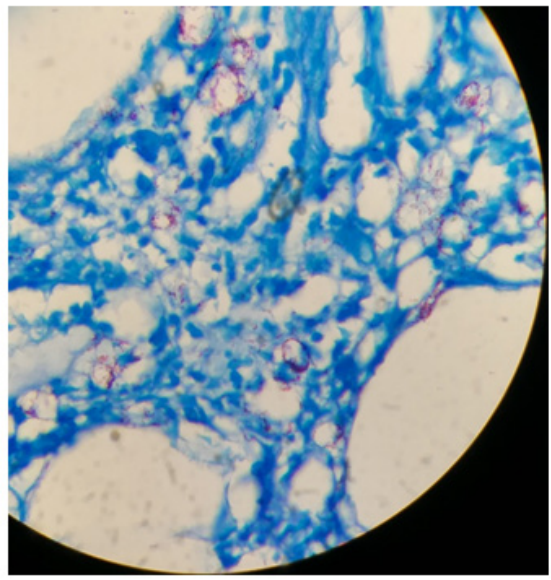

Figure 3: Zeihl-Neelson staining showing Acid Fast bacilli (Mycobacterium leprae) High- power. 
Levels of serum testosterone, luteinizing hormone ( $\mathrm{LH}$ ), folliclestimulating hormone (FSH) were 184.7ng/dl (249-836), 19.39IU/l (1.5-9.3), 23.13IU/l (1.4-18.1) respectively. The patient refused for semen analysis. He was started on multibacillary multi-drug therapy (MB MDT). Three to four days later he developed Erythema Nodosum Leprosum (ENL) which was managed with continued MB MDT and steroids. He improved and later discharged on MDT.

\section{Discussion}

Epididymo-orchitis, scrotalgia and orchitis are common urological ailments. Testicular involvement by leprosy is quite common, incidence ranging from $23.6 \%$ to $68.3 \%$ [2,3]. India accounts for half of the leprosy burden of the world. The average national child leprosy rate in India is $9 \%$ and borderline tuberculoid leprosy was the most common clinical type [5]. Trojian and colleagues have reviewed chronic epididymitis and orchitis without mention of leprosy as a differential [6]. Leprosy secondarily involves eyes, bones, lymph nodes and testes. Testicular involvement is most common, often bilateral and bloodborne. It affects both seminiferous tubules and interstitium leading to a rise in serum LH, FSH. The testes become small, firm with loss of sensation in advanced disease. Degenerated nerve fibres seen on histopathology explain diminished testicular sensation on palpation. The lower temperature of the organ is a proposed factor for the growth of M. leprae in testis [7]. Bilateral testicular atrophy results into loss of libido, infertility and impotence. Leydig cell degeneration along with liver changes could account for gynecomastia as liver takes an active part in inactivation of oestrogen. Liver biopsies may be done to document lepromata. Presence of bacilli in histopathology in Ziehl-Neelson smears is diagnostic as seen in our case. These bacilli are not seen if patients are on long term treatment [2]. Epididymal involvement in leprosy is uncommon, non-sexual and non-venereal in origin. Involvement is secondary to testis due to anatomical proximity and common vascular supply [8]. Infertility in leprosy is mainly due to the involvement of testis (85\%) with epididymis being the cause in $10-15 \%$ [7]. On the contrary, infertility in tuberculosis results from epididymal obstruction.

\section{Conclusion}

While evaluating a case of testicular atrophy, chronic Epididymoorchitis, infertility, ED or gynecomastia leprosy should be kept in mind in endemic areas. Urology textbooks do not consider leprosy as a differential diagnosis.

\section{Acknowledgment}

None.

\section{Conflict of Interest}

No conflict of interest.

\section{References}

1. Partin AW, Dmochowski RR, Kavoussi LR, Peters CA editors (2020) Campbell-Walsh-Wein Urology. 12th ed. Philadelphia: Elsevier.

2. El-Beheiry A, Zeid SA, El-Ghazzawi E, El-Mansy E, Salama N (1979) The leprous testis. Arch Androl 3(2): 173-176.

3. Migam P, Mukhija R, Gupta AK, Dayal SG, Goyal BM (1984) Gonadal Involvment In Leprosy - Study of Gynaecomastia, Testicular And Epididymal Involvement And Therapeutic Efficacy Of Indigenous Drugs. Hansen Int 9(1-2): 10-20.

4. Ridley DS, Jopling WH (1966) Classification of leprosy according to immunity. A five-group system. Int J Lepr Mycobact Dis Off Organ Int Lepr Assoc 34(3): 255-273.

5. NLEP-Annual Report for the year 2015-16, Central Leprosy Division Directorate General of Health Services Ministry of Health and Family Welfare Government of India Nirman Bhawan, New Delhi - 110011.

6. Trojian TH, Lishnak TS, Heiman D (2009) Epididymitis and Orchitis: An Overview. AmFamPhysician 79(7): 583-587.

7. Ibrahiem AA, Awad HA, Metawi BA, Hamada TAY (1979) Pathologic Changes in Testis and Epididymis of Infertile Leprotic Males'. International Journal of Leprosy 47(1): 44-49.

8. Tilak CT (1968) Acute Epididymo-Orchitis in Lepromatous Leprosy. Lepr Rev 39: 31-36. 